\title{
Can Froissart bound explain hadron cross-sections at high energies?
}

\author{
Anatoly Dymarsky \\ Skolkovo Institute of Science and Technology, \\ Novaya St. 100, Skolkovo, Moscow Region, 143025 Russia \\ E-mail: dymarsky@skoltech.ru
}

ABSTRACT: Experimentally observed slow growth of hadron cross-sections at high energies is a very intriguing but poorly understood property of QCD. It is tempting to explain the slow growth by saturation of Froissart bound or another similar universal mechanism. We reconsider derivation of Froissart bound in QCD in chiral limit and argue it can not justify experimentally observed behavior. Although the conventional Froissart-Martin bound should impose non-trivial constraint on the growth of hadron cross-sections, because of the small value of pion masses it will become restrictive only at currently unaccessible center-of-mass energies exceeding $10^{5}-10^{6} \mathrm{GeV}$.

KEYwords: QCD, Sum Rules, Nonperturbative Effects, Chiral Lagrangians

ARXIV EPRINT: 1412.8642 


\section{Contents}

1 Introduction 1

2 Froissart bound revisited 3

2.1 Froissart bound in chiral limit 5

$\begin{array}{lll}3 & \text { Summary } & 6\end{array}$

$\begin{array}{lll}\text { A Legendre functions of the second kind } & 8\end{array}$

\section{Introduction}

Experimentally observed slow growth of hadron cross-sections at high energies is an intriguing phenomenon calling for an explanation within the framework of quantum chromodynamics. For concreteness in this paper we focus on total cross-section of proton-proton scattering. It changes only about 2.5 times, roughly from $40 \mathrm{mb}$ to $120 \mathrm{mb}$, while the center-of-mass energy changes by several orders of magnitude from 10 to $10^{5} \mathrm{GeV}[1,2]$, see figure 1. This very slow growth can be approximated with a good accuracy for the center-of-mass energies exceeding $100 \mathrm{GeV}$ with the following fit (see [1, 2]),

$$
\sigma_{p p} \simeq 34.71+0.2647 \log ^{2}\left(\frac{s}{16}\right)
$$

Here $\sigma_{p p}$ is a total proton-proton cross-section measured in mb, while the Mandelstam variable $s$ is measured in $\mathrm{GeV}^{2}$. Other hadron cross-sections, e.g. of proton-anti-proton scattering, exhibit similar slow growth behavior $[1,2]$.

Because the theory in question is strongly coupled, high-energy behavior of $\sigma_{p p}$ can not be immediately deduced from QCD dynamics. At the same time "logarithm square" growth of (1.1) looks temptingly similar to the behavior of the upper bound on the total cross-section proposed by Froissart in [3],

$$
\sigma_{F}=\sigma_{0} \log ^{2}\left(\frac{s}{s_{0}}\right)
$$

The constant $\sigma_{0}$ is inverse-proportional to the nearest location of the branch-cut singularity in $t$-channel, $\sigma_{0} \sim 1 / t_{0}$, and $s_{0}$ is some appropriate constant. Any total cross-section $\sigma$ in a given gapped theory may not exceed $\sigma_{F}$ when energy $s$ is sufficiently large. It looks deceptively simple to declare the particular form of (1.1) to be a consequence of saturation of Froissart bound (1.2).

In the context of QCD the constant $\sigma_{0}$ was fixed in $[4,5]$ by Martin and Eukaszuk leading to Froissart-Martin bound $\sigma_{0}^{\mathrm{FM}}=\pi / m_{\pi}^{2} \simeq 63.32 \mathrm{mb}$. (The value of $s_{0}$ was recently 


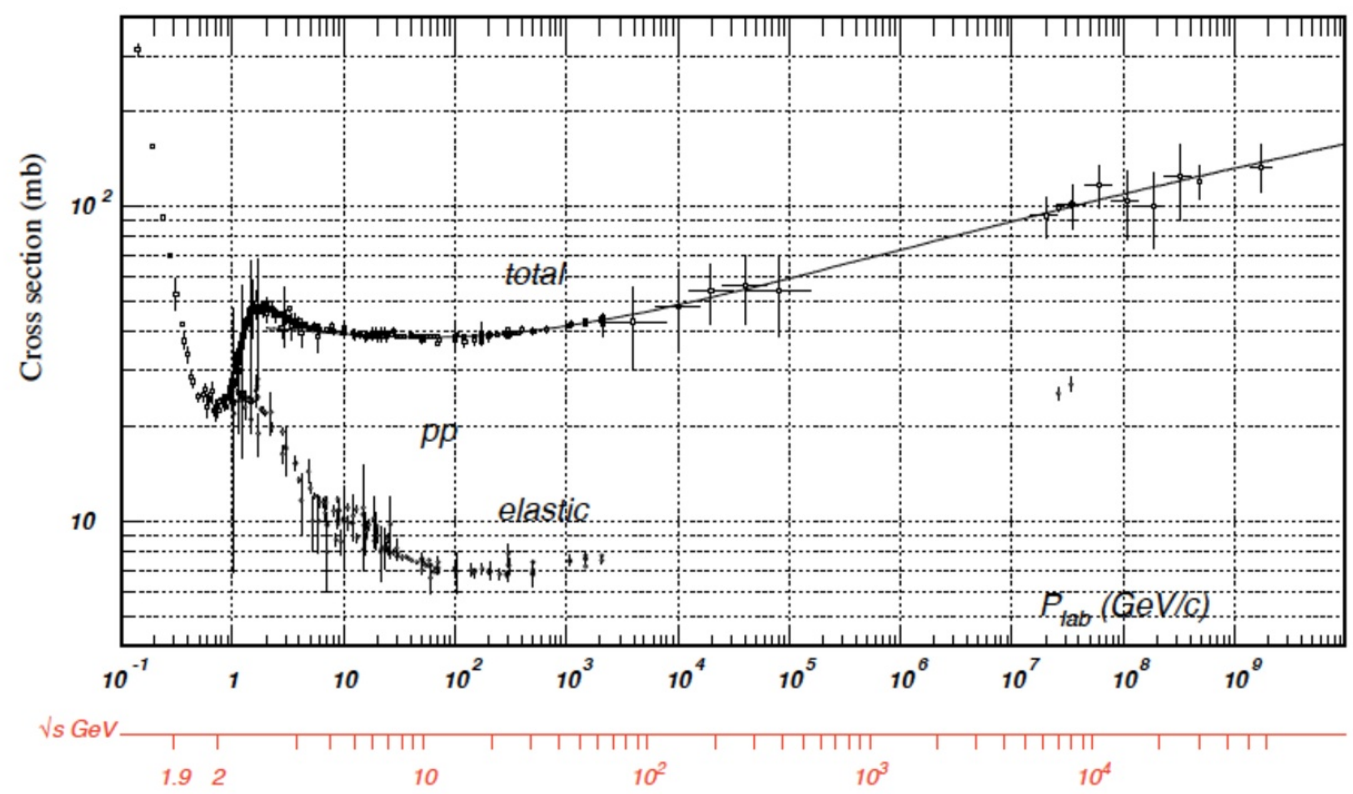

Figure 1. Proton-proton total cross-section as a function of center-of-mass energy $\sqrt{s}$. The plot is taken from $[1,2]$.

fixed to be $s_{0}=m_{\pi}^{2} \sqrt{2} /\left(17 \pi^{3 / 2}\right)[6]$, see also [7, 8] for related progress.) The two orders of magnitude difference between $\sigma_{0}^{\mathrm{FM}}$ and the coefficient in front of $\log ^{2}(s)$ in (1.1) is a clear indication that Froissart-Martin bound is currently not saturated and thus can not justify experimentally observed behavior of $\sigma_{p p}$.

Several authors suggested that the huge discrepancy between the actually observed slope of $\log ^{2}(s)$ in (1.1) and the prediction of the Froissart-Martin bound $\sigma_{0}^{\mathrm{FM}}$ is due to pseudo-Goldstone nature of pions. In chiral limit $m_{\pi} \rightarrow 0$ naive Froissart bound becomes obsolete, but it was argued there is another improved bound of the form (1.2) with finite value of $\sigma_{0}$. Since large impact parameters correspond to a small invariant mass of the pion pair, and low energy Goldstone bosons decouple, it was argued in $[9,10]$ that $\sigma_{0}$ would be determined by the strong interaction scale of $M \sim 1 \mathrm{GeV}, \sigma_{0} \sim 1 / M^{2}$. A similar conclusion was also reached in $[11,12]$ and in $[13,14]$, where $\sigma_{0}$ was calculated to be given by some combination of $\rho$-meson mass and $f_{\pi}$.

In section 2 we reconsider derivation of Froissart bound in presence of massless or very light (pseudo)-Goldstone bosons and conclude that the asymptotic form (1.2) with $\sigma_{0} \sim 1 / M^{2}, M \sim 1 \mathrm{GeV}$ is unwarranted. Furthermore, even if the resulting value of $\sigma_{0}$ would approximately match the coefficient in front of $\log ^{2}(s)$ from (1.1), still the behavior of (1.1) for $10^{2}<\sqrt{s}<10^{5} \mathrm{GeV}$ could not be justified by saturation of (1.2). This is because the bound (1.2), if holds, has to apply whenever $\sigma_{p p}(s) \gg \sigma_{0}$ (this point is justified in section 2 below), but presence of the constant term in (1.1) precludes saturation of (1.2) for $10^{2}<\sqrt{s}<10^{5} \mathrm{GeV}$. We further elaborate on this point in Summary.

To summarize, our first conclusion is that the slow growth of the proton-proton total cross-section experimentally observed for energies smaller than $10^{5} \mathrm{GeV}$ can not be at- 
tributed to saturation of the appropriately modified Froissart bound. Still, it would be interesting to understand if a similar logic can help predict the asymptotic behavior of proton-proton total cross-section when $s \rightarrow \infty$. Let us note that we can not take for granted that the form (1.1) will persist much longer beyond currently accessible scales. Indeed there is some evidence $\sigma_{p p}$ may grow faster than the "logarithm square" growth of (1.1) [15-20]. This conclusion is in agreement with an alternative fit of experimental data [21-23],

$$
\sigma_{p p} \simeq 21.7 s^{0.0808}
$$

which agrees well with (1.1) for $10^{2}<\sqrt{s}<10^{6} \mathrm{GeV}$ but grows much faster when $s \rightarrow \infty$. Clearly (1.3) would start violating (1.2) with any $\sigma_{0}, s_{0}$ when $s$ is significantly large. ${ }^{1}$ As was mentioned above, if Froissart-Martin bound holds, it must apply when $\sigma_{p p}$ becomes significantly larger than $\sim 2 \sigma_{0}^{\mathrm{FM}} \simeq 130 \mathrm{mb}$ which is expected to happen at energies of order $\sqrt{s} \sim 10^{5}-10^{6} \mathrm{GeV}$. Obviously we can not say if $\sigma_{p p}$ will eventually saturate the bound but we expect that $\sigma_{p p}$ will exhibit its true asymptotic behavior starting at or below this scale.

\section{Froissart bound revisited}

Let us briefly remind the reader main steps leading to the derivation of Froissart bound. We consider scattering of two identical particles at center-of-mass energies $\sqrt{s}$ much larger than their mass. Using optical theorem the total cross-section can be expressed through the imaginary part of forward scattering amplitude

$$
\sigma_{\text {total }}=\frac{4 \pi}{s} \operatorname{Im} A(s, \cos \theta=1) .
$$

The $2 \rightarrow 2$ elastic scattering amplitude $A(s, \cos \theta)$ can be decomposed into partial waves with help of the Legendre polynomials $P_{l}$,

$$
A(s, \cos \theta)=\sum_{l=0}^{\infty} a_{l}(s)(2 l+1) P_{l}(\cos \theta) .
$$

The upper bound on (2.1) is a result of the inequality $\operatorname{Im} a_{l} \leq\left|a_{l}(s)\right|<1$, which is a consequence of unitarity, and a bound on $\operatorname{Im} a_{l} \leq\left|a_{l}(s)\right|$ for large $l$ following from analyticity of $A(s, x)$ with respect to $x$. Rewriting $x$ through Mandelstam variables $x=$ $\left(1+\frac{2 t}{s}\right)$ we conclude $A(s, x)$ as a function of $x$ must have branch-cuts along real axis for $|x|>z_{0}$ where $z_{0}=1+2 t_{0} / s$ and $t_{0}$ is the energy squared of the lightest state appearing in $t$-channel. Assuming these branch-cuts are the only singularities of $A(s, x),{ }^{2}$ one can

\footnotetext{
${ }^{1}$ Here we implicitly assume that scattering amplitude as a function of energy grows not faster than a polynomial. This assumption may be unjustified allowing $\sigma \sim s^{\alpha}$ at all energies [15, 16, 24].

${ }^{2}$ This is a technical assumption which we believe can be avoided. Thus, [24] derives Froissart bound using only analyticity of $A$ inside Lehman ellipse, without making any explicit assumptions about structure of the singularities of $A(s, x)$.
} 
express $A(s, x)$ using dispersion relation

$$
\begin{aligned}
A(s, x) & =\int_{z_{0}}^{\infty} \frac{d z}{z-x} \rho(s, z), \\
2 i \pi \rho(s, z) & =A(s, z+i \epsilon)-A(s, z-i \epsilon)+A(s,-z-i \epsilon)-A(s,-z+i \epsilon) .
\end{aligned}
$$

The dispersion relation (2.3) may be divergent. In such a case corresponding integral should be regularized by a number of subtractions. Consequently $a_{l}(s)$ can be expressed through the branch-cut jump function $\rho$ and Legendre function of the second kind $Q_{l}(z)$ as follows (for $l$ large enough this integral is convergent and does not require subtractions)

$$
a_{l}(s)=\int_{z_{0}}^{\infty} Q_{l}(z) \rho(s, z) .
$$

Using asymptotic from of $Q_{l}(z)$ for large $l$ derived in appendix this integral can be estimated from above with help of Laplace method. First, let us assume $|\rho(s, z)|$ near $z \rightarrow 1^{+}$can be approximated, or bounded from above, by some function $\mathcal{A}(s),|\rho(s, z)| \leq \mathcal{A}(s)$. Then

$$
\begin{aligned}
& a_{l}(s) \equiv \mathcal{A}(s) \int_{z_{0}}^{\infty} Q_{l}(z) \lesssim \mathcal{A}(s) \begin{cases}\frac{1}{l^{2}}, & z_{0}-1 \ll \frac{1}{8 l^{2}} \\
\frac{\sqrt{\pi} \sqrt{1-e^{-2 \alpha_{0}}}}{2 l^{3 / 2}} e^{-\alpha_{0} l}, & z_{0}-1 \gtrsim \frac{1}{8 l^{2}}\end{cases} \\
&\left|a_{l}(s)\right| \leq \mathrm{a}_{l}(s) .
\end{aligned}
$$

Integral (2.5) is a monotonically deceasing function of $l$. Starting from some $l=L$ constraint (2.6) will become better than the unitarity constraint $\left|a_{l}(s)\right|<1$. Following [24] we introduce the combination $Y=\alpha_{0} L$, where

$$
\alpha_{0}=\log \left(z_{0}+\sqrt{z_{0}^{2}-1}\right)
$$

and notice that in the limit $t_{0} / s \rightarrow 0$ conditions $Y \gg 1$ and $z_{0}-1 \gg 1 / L^{2}$ are the same.

Assuming that $\mathcal{A}(s)$ grows with $s$, at some point $\mathcal{A}(s)$ will become large enough such that corresponding $Y \sim \log \mathcal{A}(s) \gg 1$. To find a bound on (2.1) we split the sum (2.2) into two parts, from 0 to $L-1$ and from $L$ to infinity and use unitarity bound $\left|a_{l}\right| \leq 1$ and (2.6) correspondingly. Keeping only leading terms we arrive at (see e.g. [24] for a similar calculation)

$$
\sigma_{\text {total }} \leq \frac{4 \pi}{s}\left(L^{2}+2 L / \alpha_{0}-1 / \alpha_{0}^{2}\right)=\sigma_{0}\left((Y+1)^{2}-2\right), \quad \sigma_{0} \equiv \frac{\pi}{t_{0}} .
$$

Notice that by assumption $Y \gg 1$ and hence (2.8) is always positive. Common lore predicts $\mathcal{A}(s) \sim s^{N}$ for large $s$, leading to $\sigma_{0}=\pi N^{2} / t_{0}$ and asymptotic form (1.1). Taking $\mathcal{A}(s) \sim s^{2}$ and $t_{0}=4 m_{\pi}^{2}$ we recover the Froissart-Martin bound for hadron scattering ${ }^{3}$

$$
\sigma_{\text {total }} \lesssim \frac{\pi}{m_{\pi}^{2}} \log ^{2}\left(s / s_{0}\right)
$$

\footnotetext{
${ }^{3}$ Strictly speaking the lightest singularity in $t$-channel is a one pion pole at $t_{0}=m_{\pi}^{2}$. It can be shown though that the contribution of pole singularities goes to zero when $s \rightarrow \infty$, see [24]. Hence the leading singularity contributing to the Froissart bound is a two-pion state $t_{0}=4 m_{\pi}^{2}$.
} 
Different assumptions about $\rho$ may lead to different $1 / l$ suppression of $a_{l}$ for large $l \gg \alpha_{0}^{-1}$ which will result in different $Y$-independent term in (2.8). In any case this constant term is always of order 1 and can not justify the asymptotic form $\sigma_{F}=\sigma_{0} \log ^{2}\left(s / s_{0}\right)+\sigma_{1}$ with $\sigma_{0}$ and $\sigma_{1}$ being different by several orders of magnitude as in (1.1).

An interesting but rarely discussed possibility is when $\sqrt{s}$ is much larger than the masses of external particles and $\sqrt{t_{0}}$, but $\mathcal{A}(s)$ is not too large such that corresponding $Y$ is of order or smaller than one. Then for $l \leq L, \mathrm{a}_{l}=\mathcal{A}(s) / l^{2}$ and $L \simeq \mathcal{A}^{1 / 2}(s)$. Once again we split the sum (2.6) into two parts, but now the sum from $l=L$ to infinity is tricky. It starts as $\sim \sum(2 l+1) / l^{2}$ for $L<l \lesssim \alpha_{0}^{-1}$ and continues as $\sim \sum(2 l+1) / l^{3 / 2} e^{-\alpha_{0} l}$ for

$\alpha_{0} l \gtrsim 1$. Up to double-log corrections the first sum for $L<l \lesssim \alpha_{0}^{-1}$ can be estimated as $-2 \log \left(\alpha_{0} L\right)$ while the second one gives an order one constant $\gamma$. Eventually we find

$$
\sigma_{\text {total }} \leq \sigma_{0} Y^{2}(1-2 \log (Y)+\gamma), \quad Y=\alpha_{0} \mathcal{A}(s)^{1 / 2} \ll 1
$$

The form of (2.10) is different from the canonical "log-squared" form (1.2). If $\mathcal{A}(s)$ grows polynomially, so is $Y$, while (2.10) is a combination of polynomial and logarithmic growth. For example, taking $\mathcal{A}(s) \sim s^{2}$ we get $Y \sim \sqrt{s}$ and (2.10) will be growing as $s \log (s)$. We have to conclude there is no natural reason for (2.10) to grow as a very small power of $s$ reproducing (1.3) or exhibiting a similar behavior.

Let us now discuss the area of validity of (2.8) and (2.10). The bound (2.10) is valid when $Y \ll 1$ and consequently when (2.10) is much smaller than $\sigma_{0}$. The bound (2.8) is valid when $1 / Y$ corrections are small compared with $(Y+1)^{2}$, i.e. approximately starting from $Y \gtrsim 1$, and correspondingly when $(2.8)$ is larger than $\sim 2 \sigma_{0}$. Obviously validity of (2.8) improves with growth of $Y$. When $Y \sim 1$ the bound on the total cross section is of order $\sigma_{0}$.

\subsection{Froissart bound in chiral limit}

One line of thought suggests the Froissart-Martin bound (2.9) is giving unrealistically high values for proton-proton total cross-section because pions, responsible for the lightest state appearing in $t$-channel, are pseudo-Goldstone bosons. Taking this logic to extreme it would be interesting to see what happens with the Froissart bound in chiral limit $m_{\pi} \rightarrow 0$. Naively in such a case $\sigma_{0} \sim 1 / t_{0}$ becomes infinite and Froissart bound becomes obsolete. Some authors suggested there is another improved bound of the form (1.2) with finite value of $\sigma_{0}$. Since large impact parameters correspond to a small invariant mass of the pion pair, and low energy Goldstone bosons decouple, it was argued in $[9,10]$ that $\sigma_{0}$ would be determined by the strong interaction scale of $\sim 1 \mathrm{GeV}$. A similar conclusion was also reached in [11-14].

Below we consider Froissart bound in QCD in chiral limit but reach different conclusions. Because of the Goldstone mechanism origin, at low energies pions interact through derivative couplings. Hence for small physical $s$ and $t$ amplitude $A$ is small, $A \rightarrow 0$ when $s, t \rightarrow 0$. By extrapolating this property to unphysical region, we assume the jump on the branch-cut $\rho$ vanishes when $t \rightarrow 0$ and $s$ is large an physical. In other words, we assume $|\rho(s, z)| \lesssim \mathcal{A}(s)(z-1)^{a}$ for some $a \geq 1$ at the vicinity of $z \rightarrow 1^{+}$. 
The following analysis is essentially the same for any $a$ and for convenience we fix $a=1$ unless noted otherwise. Using (A.10) and (A.11) we define

$$
a_{l} \equiv \mathcal{A}(s) \int_{z_{0}}^{\infty} d z(z-1) Q_{l}(z) \lesssim \mathcal{A}(s) \begin{cases}\frac{2}{l^{4}}, & z_{0}-1 \ll \frac{25}{8 l^{2}} \\ \frac{\sqrt{\pi}\left(1-e^{\left.-\alpha_{0}\right)^{2+1 / 2}}\right.}{2 \sqrt{2} l^{3 / 2}} e^{-\alpha_{0} l}, & z_{0}-1 \gtrsim \frac{25}{8 l^{2}}\end{cases}
$$

such that $\left|a_{l}(s)\right|<a_{l}(s)$. Up to an $l$-independent pre-factor, for $l^{2}\left(z_{0}-1\right) \gg 1$ integral (2.11) is the same as (2.5) for any $a$. Hence, when $\mathcal{A}(s)$ is large enough and $Y \sim \log \mathcal{A}(s) \gtrsim 1$ the upper bound will be also given by (2.8). When $Y \ll 1$ the situation is different. In this case $L=2^{-1 / 4} \mathcal{A}^{1 / 4}(s)$ (more generally $L \sim \mathcal{A}^{1 /(2 a+2)}(s)$ ) and as before we split the sum (2.2) into two parts. First part, from $l=0$ to $L-1$ yields $L^{2}$, while the second part from $l=L$ to infinity is bound by $\sim \sum_{l \geq L}(2 l+1) / l^{4}$ and converges to $\sim 1 / L^{2}$. Hence

$$
\sigma_{\text {total }} \lesssim 2 \sigma_{0} Y^{2}, \quad Y=2^{-1 / 4} \alpha_{0} \mathcal{A}(s)^{1 / 4} \ll 1 .
$$

For general $a, \sigma_{\text {total }} \lesssim \sigma_{0} Y^{2}\left(1+\frac{1}{a}\right)$ and $Y \sim \alpha_{0} \mathcal{A}(s)^{1 / 2(1+a)}$.

Once again we notice that the asymptotic form (2.8) corresponding to $Y \gtrsim 1$ is valid when $\sigma \gtrsim 2 \sigma_{0}$ while the asymptotic form corresponding to $Y \lesssim 1$ is valid when $\sigma \lesssim 2 \sigma_{0}$.

The bound (2.12) remains finite in chiral limit $t_{0} \rightarrow 0$,

$$
\sigma_{\text {total }}<c \frac{\mathcal{A}(s)^{1 /(1+a)}}{s},
$$

where $c$ is an appropriate numerical coefficient. Assuming $\mathcal{A}(s) \sim s^{N}, N>(1+a)$, we arrive at one of the main conclusions of this paper: in chiral limit when pions are massless or when the observed $\sigma \lesssim \sigma_{0}$, Froissart bound may have a "power law" rather than "log squared" form. The "power law" asymptotic form of $\sigma$ as a function of $s$ is corroborated by a perturbative consideration of QCD evolution equation [25, 26], although some authors believe arguments based on gluon saturation should lead to the conventional "log squared" asymptotic [27]. Even though the possibility of the power law growth of the bound is remarkable, it can hardly explain experimentally observed slow growth of (1.3) through saturation. Indeed, small power of $s$ in (2.13) would require either fine-tuning of $N$ and $a$ to some fractional values, or unrealistically large $a$.

\section{Summary}

This paper is devoted to the question whether Froissart bound can explain experimentally observed slow growth of hadron total cross sections at high energies, in particular total cross-section of proton-proton collisions. To this end we revisited derivation of Froissart bound, in particular considered what happens with the bound in chiral limit, when pions become massless. By making an appropriate assumption about the behavior of function $\rho$ (jump of the scattering amplitude across the cut in $t$-plane) we derived new universal expression for the Froissart bound (2.13) which should be valid in theories with (pseudo)Goldstone bosons while the cross-section $\sigma \ll \sigma_{0}=\pi / t_{0}$. 
There are several findings which we believe have universal nature relevant for any QFT and do not depend on technical assumptions about analytic structure of scattering amplitude, e.g. validity of dispersion relation. Applying these results toward hadron scattering lead us to several interesting conclusions. Below we summarize our results.

- In theories with mass gap there are several different regimes corresponding to different functional forms of Froissart bound. If $t_{0}$ is the lightest state appearing in $t$-channel, with an exception of bound states and stable particles, and $\sigma_{0}=\pi / t_{0}$, and the total cross-section $\sigma$ is significantly larger than $2 \sigma_{0}$, Froissart bound assumes its conventional "log squared" form (1.2), (2.8), provided scattering amplitude grows with energy as a polynomial $\mathcal{A}(s) \sim s^{N}$. When $\sigma_{F} \lesssim \sigma_{0}$, functional form of Froissart bound may vary, but the common element is the power law growth of the bound with energy when $\sigma_{F} \ll \sigma_{0}$.

In short, $\sigma \gtrsim 2 \sigma_{0}$ is an approximate validity condition of the bound $\sigma<\sigma_{0} \log ^{2}\left(s / s_{0}\right)$.

- In theories with massless Goldstone bosons, e.g. QCD in chiral limit, Froissart bound will grow with energy polynomially (2.13). This is a hypothesis based on the assumption of the particular behavior of the branch-cut jump function $\rho$ when Goldstones are the only massless states present in the theory.

- In cases when Froissart bound grows with energy polynomially or approximately polynomially, e.g. in theories with (pseudo)-Goldstones when the total cross-section is much smaller than $\sigma_{0}$, we are unaware of a mechanism which would favor small power of polynomial growth. In the context of proton-proton total cross-section we conclude that the approximate empirical power-law growth with small scaling exponent in the energy range $10^{2} \sqrt{s}<10^{5} \mathrm{GeV}$, (1.3), can not be explained by saturation of the modification of the Froissart-Martin bound in the regime $\sigma_{p p} \lesssim \sigma_{0}^{\mathrm{FM}}=63.32 \mathrm{mb}$.

- We equally conclude that the approximate empirical "log squared" growth of the proton-proton total cross-section (1.1) in the range $10^{2} \sqrt{s}<10^{5} \mathrm{GeV}$ can not be explained by saturation of the Froissart bound (1.2) with $\sigma_{0}$ tuned to be of order of strong scale $\sigma_{0} \sim 1 / M^{2}, M \sim 1 \mathrm{GeV}$, to match the coefficient in front of $\log ^{2}(s)$ from (1.1). Indeed, empirical expression (1.1) includes $s$-independent constant term $34.71 \mathrm{mb}$ which is either dominant or of the same order as $0.2647 \log ^{2}(s / 16)$ for all energies within the range $10^{2}<\sqrt{s}<10^{5} \mathrm{GeV}$. As a result the proton-proton total cross-section is of order $40 \mathrm{mb}$ or larger, for $\sqrt{s}>10 \mathrm{GeV}$, i.e. several orders of magnitude larger than the proposed value of $\sigma_{0} \sim 0.2647 \mathrm{mb}$. Consequently, for all $\sqrt{s}>10$ conventional Froissart bound (1.2) with $\sigma_{0} \sim 0.2647 \mathrm{mb}$ must apply and correspondingly $\sqrt{s_{0}}$ must be of order $20 \mathrm{MeV}$ of smaller. This is three orders of magnitude smaller than the corresponding coefficient from (1.1). As a result (1.1) will be significantly smaller than (1.2) for all $10^{2}<\sqrt{s}<10^{5} \mathrm{GeV}$, thus defying the whole idea that (1.1) saturates (1.2) in this range.

- Finally, we have to conclude that experimentally observed slow growth behavior of $\sigma_{p p}$ for $10^{2}<\sqrt{s}<10^{5} \mathrm{GeV}$ can not be attributed to saturation of Froissar bound. 
It is an open question what is the true asymptotic of $\sigma_{p p}$ when $s \rightarrow \infty$. It is likely that both "power law" (1.3) and "log squared" behavior (1.1) fail to capture true asymptotic of $\sigma_{p p}$. While the "power law" will ultimately violate Froissart-Martin bound, there is also some circumstantial evidence that "log squared" behavior of $\sigma_{p p}$ does not continue at very high energies [15-20]. The Froissart-Martin bound should apply once $\sigma_{p p}$ becomes significantly larger than $2 \sigma_{0}^{\mathrm{FM}} \simeq 130 \mathrm{mb}$, i.e. at energies above $10^{5}-10^{6} \mathrm{GeV}$. We do not know if $\sigma_{p p}$ will eventually saturate the bound, but we expect $\sigma_{p p}$ to start exhibiting its true asymptotic behavior starting from this scale. Our expectations is a result of dimensional analysis. For $\sqrt{s}>10^{6} \mathrm{GeV}$ there would be no other scale which could potentially interfere with the asymptotic behavior.

To conclude, we admit that currently available fits (1.1) and (1.3) may fail to predict $\sigma_{p p}$ at high energies above $\sqrt{s}>10^{6} \mathrm{GeV}$ and expect that $\sigma_{p p}$ will start exhibiting its true asymptotic behavior at or below this scale.

\section{Acknowledgments}

I would like to thank Slava Rychkov for bringing this topic to my attention. I am thankful to Ya.I. Azimov, D. Kharzeev, and M. Stephanov for helpful correspondence and gratefully acknowledge support from the grant RFBR 15-01-04217.

\section{A Legendre functions of the second kind}

Here we collect some useful relations related to the Legendre functions of the second kind $Q_{l}(z)$. We define $Q_{l}(z)$ through Legendre polynomial $P_{l}$ as follows

$$
Q_{l}(z) \equiv \frac{1}{2} \int_{-1}^{1} d x \frac{P_{l}(x)}{z-x}, \quad z>1 .
$$

To derive the asymptotic form $Q_{l}(z)$ for large $l$ we use the following integral representation

$$
Q_{l}(z)=\frac{1}{2} \int_{-\infty}^{\infty} \frac{d t}{\left(z+\sqrt{z^{2}-1} \cosh (t)\right)^{l+1}}, \quad z>1,
$$

and apply Laplace method to get

$$
\lim _{l \rightarrow \infty} Q_{l}(z) \rightarrow \sqrt{\frac{\pi}{2(l+1)}} \frac{1}{\left(z^{2}-1\right)^{1 / 4}\left(z+\sqrt{z^{2}-1}\right)^{l+1 / 2}} .
$$

This is an accurate approximation of $Q_{l}(z)$ except of a small vicinity of $z \rightarrow 1^{+}$.

Now we would like to estimate $\int_{z_{0}}^{\infty} d z Q_{l}(z)$ for different values of $z_{0}$. Using (A.3) and a change of variables $z=\cosh \alpha$ we immediately find

$$
\int_{z_{0}}^{\infty} d z Q_{l}(z) \simeq \sqrt{\frac{\pi}{2(l+1)}} \int_{\alpha_{0}}^{\infty} d \alpha e^{-(l+1 / 2) \alpha} \sinh ^{1 / 2} \alpha
$$

where $\alpha_{0}=\log \left(z_{0}+\sqrt{z_{0}^{2}-1}\right)$. There are two distinct regimes, $z_{0}<\frac{(2 l+1)}{2 \sqrt{l(l+1)}} \simeq 1+\frac{1}{8 l^{2}}$ and $z_{0}>\frac{(2 l+1)}{2 \sqrt{l(l+1)}} \simeq 1+\frac{1}{8 l^{2}}$. In the former case the peak of integrand is inside the 
interval of integration and one can once again apply Laplace method to find $z_{0}$-independent asymptotic

$$
\int_{z_{0}}^{\infty} d z Q_{l}(z) \simeq \frac{\pi}{2 \sqrt{e}} \frac{1}{l^{3 / 4}(l+1)^{1+1 / 4}}, \quad z_{0}-1 \lesssim \frac{1}{8 l^{2}},
$$

which correctly reproduces $1 / l^{2}$ behavior of the exact value for $z_{0}=1$

$$
I_{l}^{0}=\int_{1}^{\infty} d z Q_{l}(z)=\frac{1}{l(l+1)} .
$$

When $\left(z_{0}-1\right) \gtrsim \frac{1}{8 l^{2}}$ the integral (A.4) can be estimated from above as

$$
\int_{z_{0}}^{\infty} d z Q_{l}(z)<\frac{\sqrt{\pi} \sqrt{1-e^{-2 \alpha_{0}}}}{2 l \sqrt{l+1}} e^{-\alpha_{0} l}, \quad z_{0}-1 \gtrsim \frac{1}{8 l^{2}} .
$$

Similarly we can estimate $\int_{z_{0}}^{\infty} d z(z-1)^{a} Q_{l}(z)$ for some positive $a$. Using the same change of variables as in (A.4) we find

$$
\int_{z_{0}}^{\infty} d z(z-1)^{a} Q_{l}(z) \simeq \sqrt{\frac{\pi}{2(l+1)}} \int_{\alpha_{0}}^{\infty} d \alpha e^{-(l+1 / 2) \alpha}\left(2 \sinh ^{2}(\alpha / 2)\right)^{a} \sinh ^{1 / 2} \alpha .
$$

Again, there are two regimes. When $z_{0}-1 \lesssim \frac{(1+4 a)^{2}}{8 l^{2}}$ the peak of integrand of (A.8) is within the area of integration. Applying Laplace method to (A.8) yields

$$
\int_{z_{0}}^{\infty} d z(z-1)^{a} Q_{l}(z) \simeq \frac{\pi(1+4 a)^{1+2 a}}{2^{1+3 a} e^{1 / 2+2 a} l^{2(a+1)}}, \quad z_{0}-1 \lesssim \frac{(1+4 a)^{2}}{8 l^{2}}
$$

This result correctly captures $1 / l^{2(a+1)}$ behavior of $z_{0} \rightarrow 1$ limit which can be deduced for positive integer $a$ from (A.6) and the recursion relation

$$
I_{l}^{a}=\int_{1}^{\infty} d z(z-1)^{a} Q_{l}(z), \quad I_{l}^{a+1}=\frac{2(a+1)^{2}}{l(l+1)-(a+2)(a+1)} I_{l}^{a} .
$$

When $z_{0}-1 \gtrsim \frac{(1+4 a)^{2}}{8 l^{2}}$ the peak of integrand of (A.8) is outside of the area of integration, and the integral can be estimated from above as follows

$$
\int_{z_{0}}^{\infty} d z(z-1)^{a} Q_{l}(z)<\frac{\sqrt{\pi} \sqrt{1-e^{-2 \alpha_{0}}}}{2(l-a) \sqrt{l+1}} \frac{\left(1-e^{-\alpha_{0}}\right)^{2 a}}{2^{a}} e^{-\alpha_{0} l}, \quad z_{0}-1 \gtrsim \frac{(1+4 a)^{2}}{8 l^{2}} .
$$

Open Access. This article is distributed under the terms of the Creative Commons Attribution License (CC-BY 4.0), which permits any use, distribution and reproduction in any medium, provided the original author(s) and source are credited.

\section{References}

[1] Particle Data Group collaboration, K.A. Olive et al., Review of particle physics, Chin. Phys. C 38 (2014) 090001 [inSPIRE].

[2] http://pdg.lbl.gov/2014/hadronic-xsections/. 
[3] M. Froissart, Asymptotic behavior and subtractions in the Mandelstam representation, Phys. Rev. 123 (1961) 1053 [INSPIRE].

[4] A. Martin, An absolute upper bound on the pion-pion scattering amplitude, SITP-134 (1964) [INSPIRE].

[5] L. Łukaszuk and A. Martin, Absolute upper bounds for $\pi \pi$ scattering, Nuovo Cim. A 52 (1967) 122 [INSPIRE].

[6] A. Martin and S.M. Roy, Froissart bound on total cross-section without unknown constants, Phys. Rev. D 89 (2014) 045015 [arXiv:1306.5210] [InSPIRE].

[7] A. Martin, The Froissart bound for inelastic cross-sections, Phys. Rev. D 80 (2009) 065013 [arXiv: 0904.3724] [INSPIRE].

[8] T.T. Wu, A. Martin, S.M. Roy and V. Singh, An upper bound on the total inelastic cross-section as a function of the total cross-section, Phys. Rev. D 84 (2011) 025012 [arXiv: 1011.1349] [INSPIRE].

[9] D. Kharzeev and M. Stephanov, Froissart bound and chiral limit in $Q C D$, unpublished.

[10] M. Stephanov, Froissart bound and chiral limit in $Q C D$, http://conferences.fnal.gov/smallx/Thursday\%20afternoon/Stephanov.pdf.

[11] M. Giordano and E. Meggiolaro, Hadronic total cross sections at high energy and the QCD spectrum, JHEP 03 (2014) 002 [arXiv:1311.3133] [INSPIRE].

[12] M. Giordano and E. Meggiolaro, Comments on high-energy total cross sections in $Q C D$, Phys. Lett. B 744 (2015) 263 [arXiv:1411.0553] [INSPIRE].

[13] D. Greynat, E. de Rafael and G. Vulvert, Asymptotic behaviour of pion-pion total cross-sections, JHEP 03 (2014) 107 [arXiv: 1312.2881] [INSPIRE].

[14] D. Greynat and E. de Rafael, Froissart-Martin bound for $\pi \pi$ scattering in $Q C D$, Phys. Rev. D 88 (2013) 034015 [arXiv: 1305.7045] [inSPIRE].

[15] Y.I. Azimov, Froissart bounds for amplitudes and cross sections at high energies, arXiv:1204.0984 [INSPIRE].

[16] Y. Azimov, What is the real meaning of the Froissart theorem?, in Proceedings of the International Workshop HSQCD 2012, St. Petersburg Russia, 4-8 Jul 2012, V.T. Kim and L.N. Lipatov eds., pg. 22 [arXiv:1208.4304] [INSPIRE].

[17] D.A. Fagundes and M.J. Menon, Total hadronic cross section and the elastic slope: an almost model-independent connection, Nucl. Phys. A 880 (2012) 1 [arXiv:1112.5115] [INSPIRE].

[18] D.A. Fagundes, M.J. Menon and P.V.R.G. Silva, On the rise of the proton-proton cross-sections at high energies, J. Phys. G 40 (2013) 065005 [arXiv:1208.3456] [INSPIRE].

[19] M.J. Menon and P.V.R.G. Silva, An updated analysis on the rise of the hadronic total cross-section at the LHC energy region, Int. J. Mod. Phys. A 28 (2013) 1350099 [arXiv: 1212.5096] [INSPIRE].

[20] M.J. Menon and P.V.R.G. Silva, A study on analytic parametrizations for proton-proton cross-sections and asymptotia, J. Phys. G 40 (2013) 125001 [arXiv:1305.2947] [InSPIRE].

[21] P.V. Landshoff, How well can we predict the total cross section at the LHC?, AIP Conf. Proc. 1105 (2009) 236 [arXiv:0811.0260] [INSPIRE]. 
[22] A. Donnachie and P.V. Landshoff, Elastic scattering at the LHC, arXiv:1112.2485 [INSPIRE].

[23] A. Donnachie and P.V. Landshoff, $p p$ and $\bar{p} p$ total cross sections and elastic scattering, Phys. Lett. B 727 (2013) 500 [arXiv:1309.1292] [INSPIRE].

[24] Y. Azimov, How robust is the Froissart bound?, Phys. Rev. D 84 (2011) 056012 [arXiv: 1104.5314] [INSPIRE].

[25] A. Kovner and U.A. Wiedemann, Nonlinear QCD evolution: saturation without unitarization, Phys. Rev. D 66 (2002) 051502 [hep-ph/0112140] [INSPIRE].

[26] A. Kovner and U.A. Wiedemann, No Froissart bound from gluon saturation, Phys. Lett. B 551 (2003) 311 [hep-ph/0207335] [INSPIRE].

[27] E. Ferreiro, E. Iancu, K. Itakura and L. McLerran, Froissart bound from gluon saturation, Nucl. Phys. A 710 (2002) 373 [hep-ph/0206241] [INSPIRE]. 